\title{
A Comprehensive and Practical Image Enhancement Method
}

\author{
Fanglong $\mathrm{Wu}^{1,2}$, Cuiyin Liu ${ }^{1,2,3 *}$ \\ ${ }^{1}$ Faculty of Information Engineering and Automation, KunmingUniversity of Science and Technology, No.727 \\ South Jingming Rd., ChenggongDistrict, 650500, Kunming,P.R.China \\ Yunnan Key Laboratory of Computer Technology Applications, Kunming University of Science and Technology, \\ No.727 South Jingming Rd., Chenggong District,650500, Kunming, P.R.China. \\ 3 Faculty of Computer center, Kunming Universityof Science and Technology, No.727 South Jingming Rd., \\ Chenggong District,650500, Kunming, P.R.China \\ [e-mail: liucuiyin@163.com, fanglongwu.stu@gmail.com] \\ *Corresponding author: Cuiyin Liu
}

Received December 21, 2018; revised February 28, 2019; accepted March 11, 2019; published October 31, 2019

\begin{abstract}
Image enhancement is a challenging problem in the field of image processing, especially low-light color images enhancement. This paper proposed a robust and comprehensive enhancement method based several points. First, the idea of bright channel is introduced to estimate the illumination map which is used to attain the enhancing result with Retinex model, and the color constancy is keep as well. Second, in order eliminate the illumination offsets wrongly estimated, morphological closing operation is used to modify the initial estimating illumination. Furthermore, in order to avoid fabricating edges, enlarged noises and over-smoothed visual features appearing in enhancing result, a multi-scale closing operation is used. At last, in order to avoiding the haloes and artifacts presented in enhancing result caused by gradient information lost in previous step, guided filtering is introduced to deal with previous result with guided image is initial bright channel. The proposed method can get good illumination map, and attain very effective enhancing results, including dark area is enhanced with more visual features, color natural and constancy, avoiding artifacts and over-enhanced, and eliminating Incorrect light offsets.
\end{abstract}

Keywords: Image enhancement, bright channel, morphological operation, multi-scale fusion, guided filter

This research was supported by National Natural Science Foundation of China (No. 11773012). 


\section{Introduction}

Image enhancement is an important method to improve image quality and is necessary research work in low-level image processing research [1]. For lacking of light, uneven illumination, weather changes, relative motion, overexposure and other factors, the visual quality of acquisition image is reduced, and leads corresponding challenge on subsequent applications, such as image recognition, machine vision and clinical medicine, etc. Without destroying the existing visual information content, specific image processing methods are used in image enhancement to enhance the image clarity, contrast and detail features, improve vision quality, meet requirements of recognition, information reading and image segmentation in subsequent visual processing and research, which is image enhancement technique. After the processing of image enhancement, a low-quality image is transformed into a high-quality image which meets the HVS (human vision system, HVS) and effective application of image analysis and recognition. For the different low-quality images, the different enhancing process is explored and modified for use. Because of different imaging factors and different data sources, there is no possibility of generality and generalization of image enhancement algorithms. Image enhancement is still an important part of image processing field.

\subsection{Intensity image enhancement}

Image enhancement is divided into two categories according to the source image, which is intensity image enhancement, and the other is color image enhancement. Many frequent enhancing technologies of gray image have been explored and widely used, including arithmetic operation method for global pixels, including histogram equalization and normalization based on statistics, including all kinds of pyramid method based multi-resolution, including all kinds of multi-scale method based contourlet transform, etc. The above methods can also be divided into spatial and frequency domain enhancement, depending on whether frequency transformation is carried out. Especially, the spatial domain is divided into global and local enhancement $[13,14,15,16]$. Intensity transform methods employ linear mapping and non-linear, which recalculate the intensity values of pixels in the source image based on the given mathematic function which is selected for enhancing visual quality of the specified image $[17,17,19,20]$. Computation the negative of an image is a kind of intensity transform for enhancing white or grey detail embedded in dark region. Power-law transformation, logarithmic transformation works on a narrow range of gray image, which is often used to extend range of output. The visual structure in the dark area is enhanced and shown in a wide-range out image. The opposite non-linear transformation is power-law transform $\left(\mathrm{s}=\mathrm{c}^{\mathrm{r}}\right.$ ), which is also called as gamma correction and is used for monitor automatically correct $[21,22,23]$. As the gamma value decreases, the contrast of the image increases too, and detail became visible. Gamma transformation can be used to enhance dark background to make more detail visible by decreasing the value of gamma. On the contrary, it is also can be used to enhance the image with "wash-out" appearance to compress gray levels by increasing the value of gamma. In practical application, it is necessary to combine multiple transformation to construct a piecewise function, or construct complex multi-function for variety of input. Histogram equalization is an adaptive enhancement for difference contrast image by adjusting or stretching grey levels into a uniformly distributed histogram. The histogram matching is redistributed the histogram of a specified image according to a given other image [24,25,26,27].Although global approach is suitable for adjusting overall contrast, 
brightness and distribution of gray scale. However, there are differenet local areas which need to speical enhancing process. So local spatial enhancement is needed with a transformation function devised on neighborhood of pixel. The local transformation function may be called a filter, mask, kernel, template, or window, etc terminology [28,29,30,31]. The construction of the mask is used to convolved every pixel in image to enhance local visual details, including sharpening edges, denoising, deblurring and filter.

\section{2 color image enhancement}

The above methods are effective for gray image enhancement, but still have some shortcomings for color image. Global enhance method cannot balance the uneven illumination areas of one image, may lead to some details invisible in dark background, and some details exposure in bright area. Especially, the enhancing effectiveness of above method is mostly used for gray image, but not suitable for color image. Color image enhancement not only need to enhance visual details, but also consider the color constancy, which is an example of subjective constancy and a feature of the human color perception system which ensures that the perceived color of objects remains relatively constant under varying illumination conditions [2]. Color constancy principal works on the assuming that the human perceived "true color" is determined by the wavelengths of light reflected by object, although incident light contains a range of wavelengths. The concept of color constancy was described in 1971 by Edwin H.Land, and formulated "retinex theory" and explain it, which is formed from "retina" and "cortex" suggesting both eye and brain are worked simultaneously. The experiment was also demonstrated that colors have their original colors independent of different incident light. Color constancy is a desirable feature of computer vision, the normal Retinex algorithm proposed by Land are still widely used in computer vision. For different visual information in a picture to be enhanced, many algorithms have been developed for problems. In 1985, Land himself also proposed latest progress [3]. On these basis, Jobson, etc. propose the SSR(single-scale Retinex algorithm,SSR) [4]. SSR has a good effect in dealing with the problems of insufficient illumination, uneven brightness, darkness, but halo artifacts and shadows will appear very abrupt in boundary areas with relatively large differences in gray values, which is not suitable for the HVS(Human visual system, HVS). A lot of research work has been carried out for improving and modifying the Retinex algorithm to make it effective in variable enhancement problem. Qimu, etc evaluate initial illumination component by filtering image with multi-scale gauss filters [5].

The Retinex algorithm itself with improved method based on it has made progressive result in image enhancement both research and application, but its current methods still face with challenging problems to be resolved. This paper aims to survey related Retinex algorithms, analysis their insufficience, experiment them for various enhancement needs, and modified them with different aspects, and finally a robust and effective enhancement method is proposed with several aspects, including the detail information extracting, bright channel adopted, illumination map moidified with morphological closing, mutli-scale method, and image fusion. This method is effective and comprehensive for enhancing low light image. The structure of this paper is structured as follow. Section 2 discusses Retinex algorithm and its derivation. Section 3 described the proposed method of this paper in detail. In section 4, the extensive experiments are presented to illumstrate roubst and effectiveness of the proposed method in enhancement. Finally, the objective and subjective evaluation of multi-enhancement are also presented. 


\section{Related Work}

Retinex algorithm was proposed and implemented by Jobson. The purpose of image enhancement based on Retinex is to estimate the illumination $L$ from the original image $S$, decompose the $R$, eliminate the influence of illumination unevenness, and improve the visual quality, consistent with the human visual system. Its basic progress is that the image $S$ with RGB three channels is separated into three gray images $S_{R}, S_{G}$ and $S_{B}$, which are filtered by gaussian kernel respectively for evaluation of illumination light $L\left(L_{R}, L_{G}, L_{B}\right)$. At last final reflected image is obtained by the division of $S / L$. The basic principle of Retinex is given by

$$
S(x, y)=R(x, y) \cdot L(x, y)
$$

Where, $S(x, y)$ is a source image which is composed of two parts: the illumination $L(x, y)$ and reflectance image $R(x, y)$ of the object itself. The principel of the algorithm is to obtain $R(x, y)$ by estimating $L(x, y), R(x, y)$ is the result of enhancing. The estimation of $L(x, y)$ is the most important work in modle Retinex.

\section{The Comprehensive and practical Image Enhancement Method}

How to estimate illumination, the bright channel method is a normal method attained by finding the maximum of each RGB channels in every location of pixel. A simulating experiment has been processed to explain the theoretical model.

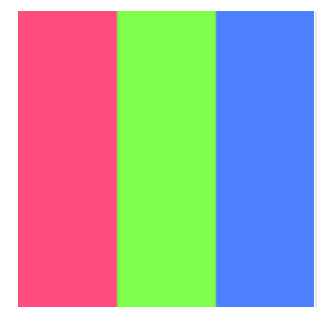

a

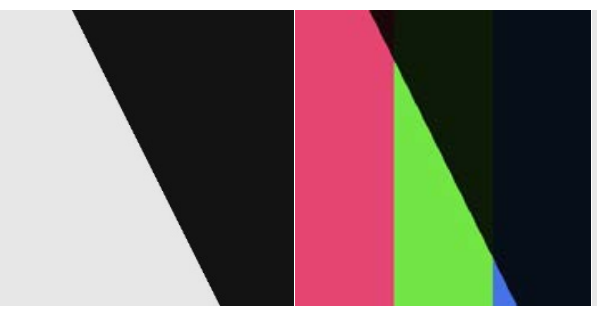

b
C

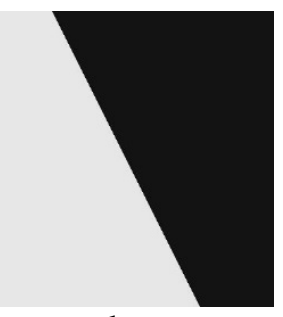

d

Fig. 1. incident light $L(x, y)$ estimating experiment. Fig. 1 (a) is simulating object being illuminated.

Fig. 1 (b) is simulation of incident light. Fig. 1 (c) is result of Fig. 1 (a) illuminated by Fig. 1 (b).

Fig. 1 (d) is estimation result of incident light by taking maximum of three channel (RGB).

Where, Fig. 1 (d) is the estimation incident light which should be similar to Fig. 1 (b) in theory. In this paper, major work is to propose an illumination estimation method bright channel estimation and define in equation (2)

$$
L_{\text {light }}=\max _{\Omega(x, y) \in(-\alpha, \alpha)}\left(L_{R G B}(x, y)\right)
$$

For the diffraction of light being exist, In order to better estimate illumination, the idea is adoped which is local area illumination intensity is recognized closely. $\Omega(x, y)$ presents the local area, and $(x, y)$ present coordination of current pixel. 


\section{1 the multi-scale morphology operation on bright channel}

There are some errors in the estimation of incident light using bright channels, such as in the illumination boundary area or local high intensity illumination area, where the estimated incident light will shift to the non-illuminated (low illumination) area compared with the real incident light. The consequence estimating value of incident light will be higher at the edge of dark area, and artifacts will be occurred in this area. In order to eliminate the deficiency, a morphology model is adopted for modifying the estimation result. The closing operator is used for correcting the problem of light shift. It is combined with dilation and erosion in orderly which is denoted by $A \cdot B$.

$$
A \cdot B=(A \oplus B) \Theta B
$$

Closing operation generally joins narrow breaks, fills thin, long, and break gaps, and fills holes smaller than the structuring element [6]. When it is used in estimation illumination with bright channel method, narrow discontinuities, over exposure, long and thin gaps and small holes will be eliminated, breaks will be filled in the contours. The simulating experiments for proving the effectiveness of closing in incident illuminstion have been carried out and the results and analysis are presented in Fig. 3.

Where, comparing Fig. 2 (b) with(c), the offset is corrected in Fig. 2(c) after closing operation. The consequence is that the black stripe in (e) has been removed in Fig. 2(f). It is obvious that closing operation has corrected the error estimaiton in incident light.

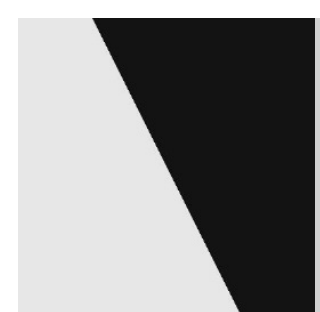

(a)

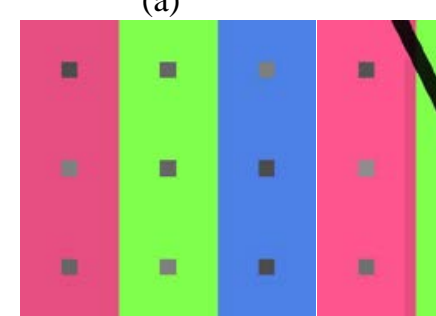

(d)

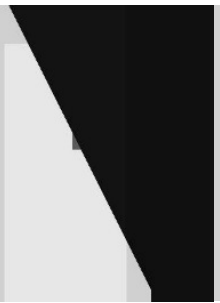

(b)

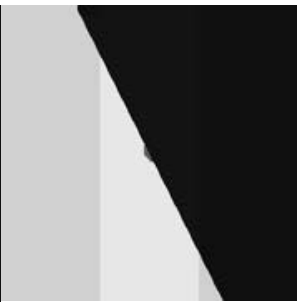

(c)

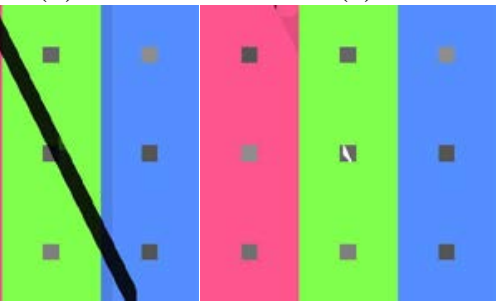

(e)

(f)

Fig. 2. the experiment of bright chanel improved by morphological closing. Fig. 2 (a) is the simulation of source illumination, (d) is the simulation object for reflecting experiment. (b) is the estimation of illumination with bight channel, (e) is the enhancing result using (b) with Retinex alorithm. (c) is the modified result of (b) with closing operation, and (f) is the enhancing result using (c) with Retinex algorithm.

A "good" solution should simultaneously preserve the overall structure and smooth the textural details [7]. To address this issue, based on the initial estimation illumination map, we proposed multi-scale closing operation to achieve the object. So far, the proposed algorithm is defined in equation (4). 


$$
L_{\text {light }} \sum_{i} \omega_{i}\left[\max _{\Omega(x, y) \in(-a, a)}\left(L_{R G B}(x, y)\right)\right] \bullet B_{i}
$$

Experiments of multi-scale closing operation used in estimation illumination map have been carried out, and the corresponding results are presented in Fig. 3.

Fig. 3(b) shows the estimation illumination, which is the bright channel processed by a closing kernel with radius of $1 \%$ of the width of source image. Using it to enhance the source image, overall brightness of the Fig. 3(e) is enhanced with an average brightness value 199.72, and more visual details are presented. However, it is still not a "good" illumination estimation in several facts, including noises enlarged, features of objects reducted and the color is not natural.

Fig. 3(c) shows the estimation illumination, which is the bright channel processed by a closing kernel with radius of $10 \%$ of the width of source image. Using it to enhance the source image, overall brightness of the Fig. 3(f) is enhanced with an average brightness value 126.82. However, it is still not a "good" illumination estimation because incorrect estimation of edges in result.

A good estimating illumination should preserve the overall structure and smooth the details structure, which are met by Fig. 3(d). Fig. 3(d) is the result of the proposed method in this paper, which is multi-scale closing operation method for illumination estimation and is obtained by fusing Fig. 3(b) and Fig. 3(c). The corresponding enhancing result is Fig. 3(g), which is natural, more visual features, and little artifacts comparing to Fig. 5(e) and (f). In this result, dark areas still have not enhanced with missing visual features is exist, which is will be resolved in next sub-section.

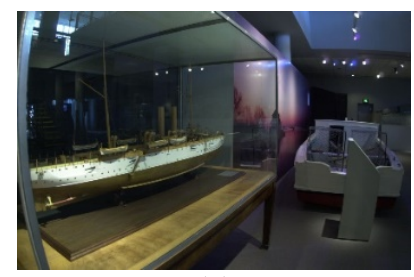

(a)

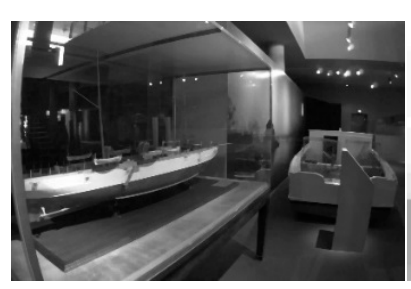

(b)

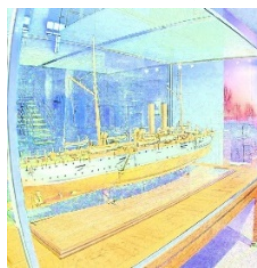

(e)

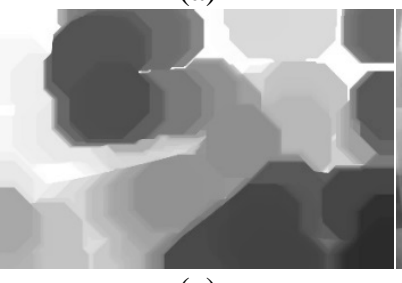

(c)

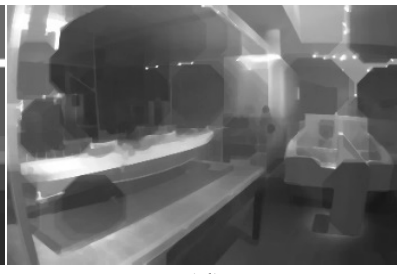

(d)

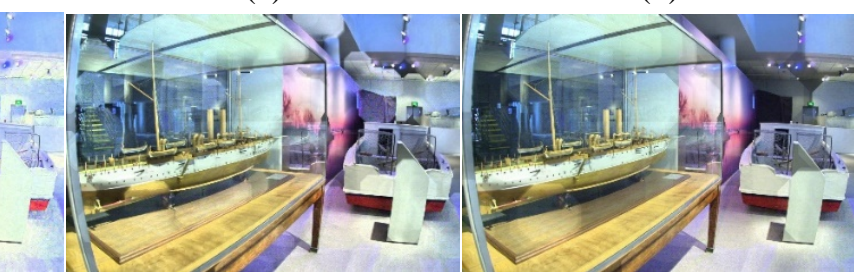

(g)

Fig. 3. Experiments of multi-scale closing operation for estimation illumination: (a) is a true low illumination image which is named source image in context, Fig. 3(b) is illumination estimation processed by smaller size of closing kernel, Fig. 3(c) is illumination estimation processed by larger size of closing kernel, Fig. 5(d) is fusion result of Fig. 3(b) and Fig. 3(c), Fig. 3(e), (f), (g) are enhancing result, which are based on Fig. 3(b), (c), (d) respectively. 


\subsection{Processing of guided filer}

In initial Retinex algorithm, using gaussian filter to estimation $L(x, y)$ which is detaied in equation (5).

$$
H\left(x_{i}, y_{j}\right)=e^{-\frac{\left(x_{i}-a\right)^{2}+\left(y_{j}-b\right)^{2}}{2 a^{2}}}, i, j \in(-n, \ldots,-1,0,1, \ldots, n)
$$

Due to the smoothing characteristics of gaussian filter, illumination information of edges from different intensity areas is smoothed, which leads to haloes and artifacts presented in the enhancement image, and underestimate the estimation value of incident light, even lower than original pixel value as well, which leads to overexposure in enhancing result.

Some modified Retinex algorithms overcomes the disadvantages of gaussian filter by using bilateral filter, which is a non-linear, edge-preserving, and noise-reducing smoothing filter for images. It calculates the intensity of each pixel not only with Euclidean distance of pixels but also with difference of intensity of pixels. The bilateral filter kernel is defined as equation (6)

$$
F(x, y, a, b)=e^{-\frac{\left\|S\left(x_{i}, y_{j}\right)-S(a, b)\right\|^{2}}{2 \sigma_{r}^{2}}-\frac{\left(x_{i}-a\right)^{2}+\left(y_{j}-b\right)^{2}}{2 \sigma 2}}, i, j \in(-n, \ldots-1,0,1, \ldots, n)
$$

The modified Retinex with bilateral filter can correct the haloes and conserve part of edges, which is corresponding to inhibite artifacts and gradient reverse in the enhacing result.

According to recent research work, the guided filter is an edge-preserving smoothing operator and has better behavior near the edges than bilateral filter. Moreover, the guided filter has a fast and an approximate linear-time algorithm, whose computational complexity is independent of the filtering kernel size[8].

The theory of guided is described as equaiotn [7]:

$$
q_{i}=\sum_{i} W_{i j}(I) p_{i}
$$

Where $\mathrm{i}$ and $\mathrm{j}$ are pixel indexes. The filter $W_{i j}$ is a function of the guidance image I and independent of p. The filter result is relative to guidance image $I$ and not relative to source image $p$. The guided filter and its kernel are defined as equation (8)

$$
q_{i}=a_{k} I_{i}+b_{k}, \forall i \in \omega_{k}
$$

Where, $\omega_{k}$ is a square window of a radius $\mathrm{r},\left(a_{k}, b_{k}\right)$ are some linear coefficients assumed to be constant in $\omega_{k}$. The edges of output image $q$ ared determined by the guidance image $I$, because $\nabla q=a \nabla I$.The parameters of $\left(a_{k}, b_{k}\right)$ are based convex mathematical model which is described and proved in paper [8].

$$
a_{k}=\frac{\frac{1}{|\omega|} \sum_{i \in \omega_{k}} I_{i} p_{i}-\mu_{k} \bar{p}_{k}}{\sigma_{k}^{2}+\varepsilon}
$$




$$
b_{k}=\bar{p}_{k}-a_{k} \mu_{k}
$$

The guided filter has nice property of edge-preserving, efficient computation and relization simple. In order to illustrate importance of guided filter in estimating illumination, a group experiments of modified Retinex algorithm have carried out. The results are present in Fig. 4.

Where, Fig. 4(b) shows the estimation illumination, which is the bright channel processed by a closing kernel with radius of $1 \%$ of the width of source image. Using it to enhance the source image, overall brightness of the Fig. 4(e) is enhanced with an average brightness value 199.72, and more visual details are presented. However, it is still not a "good" illumination estimation in several facts, including noises enlarged, features of objects reducted and the color is not nutural. Comparing Fig. 4(b) (c) (d) and (e), the difference is obvious. (b) is shoft of details and without estimation uneven light. The situation is improved in (c) a little, and is better improved in (d). The "good" illumination estimation is (e) preserved overall structures and detail structures which is refect variation of light of local area. Fig. 6(i) is the enhancing result of proposed method which is natrual in color, contranst and brightness is suitable for HVS, and more visul features are presented.

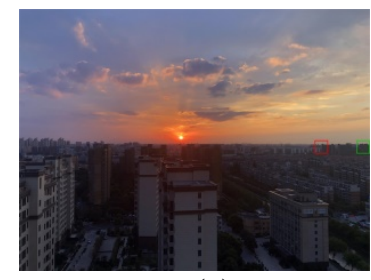

(a)

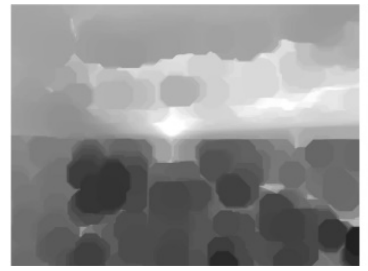

(b)

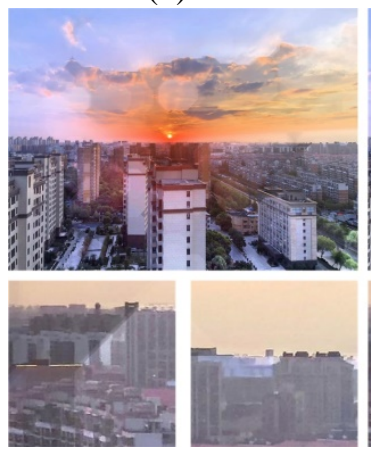

(f)

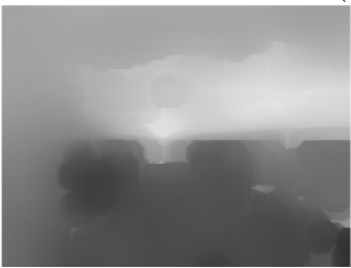

(c)

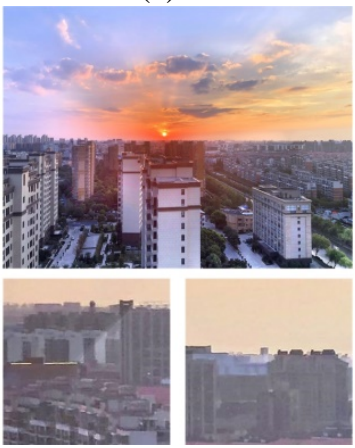

(g)

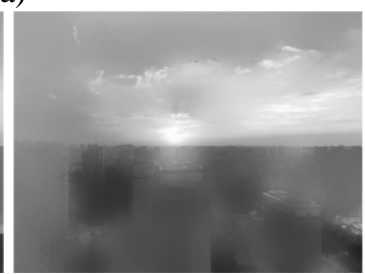

(d)

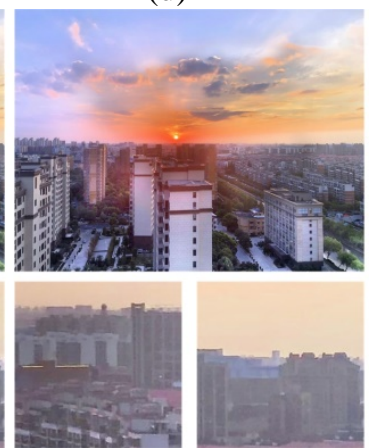

(h)

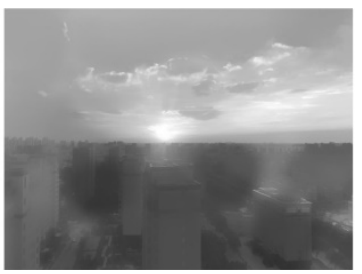

(e)

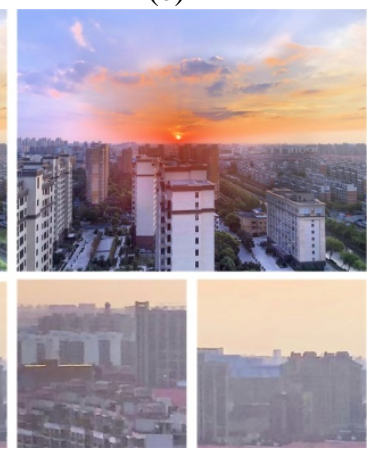

(i)

Fig. 4. The experiment of proposed method: (a) is a true low illumination image which is named source image in context. (b) is illumination estimation processed by single closing kernel. (c) is filtered result of (b) by bilateral filter. (d) is filtered result of (b) by guided filter.(e) is guided filtered result of a fuison image which is fuison two different image (b) processed by different size of closing kernel.Fig. 4(f),

Fig. 4(f), (g), (h) and (i) are enhancing result and local detailed presentation denoted in red box and green box in souce image based on Fig. 4(b), (c), (d) and (e) respectively. 


\subsection{Proposed method}

This paper present an improved method of Single-Scale Retinex. where, on the basis of the bright channel, the illumination is estimated by two different size kernel of closing operation. The estimated illumination is substantially consistent with the true illumination. Finally, use guided filter to correct and refine the edge regions of closing result. the flow chart of the proposed method is shown in Fig. 5.

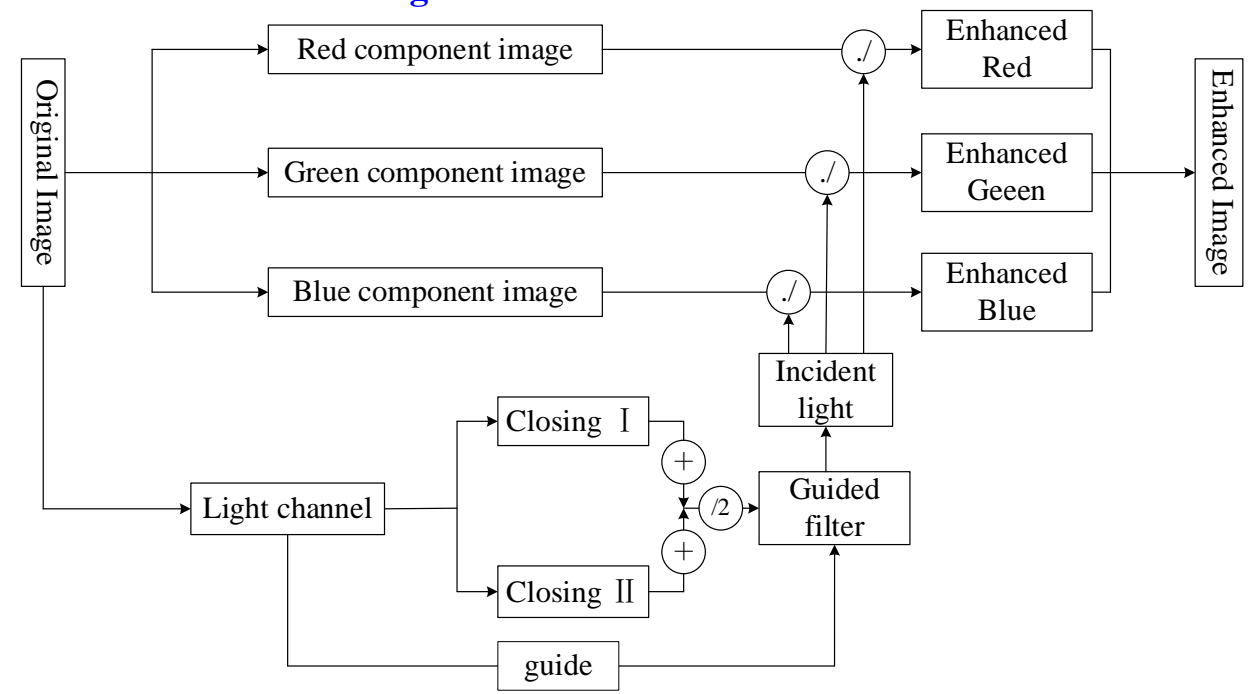

Fig. 5. Flowchart of the method in this paper

Fig. 6 shows the images of each step in the process of implementing the flowchart. The size of the source image is $1152 * 864$, the size of the rectangular neighborhood around pixel used in light channel is 864/600, in two different sizes of closing kernel are 864/100 and 864/20, in guided filter is $864 / 10$ (parameter which not an integer, it will be rounded off); The precision of guiding filter is 0.0001 .
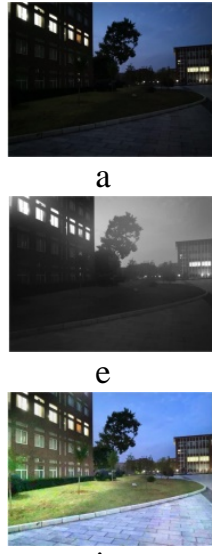

i

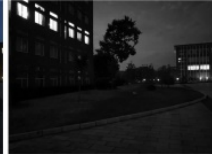

b

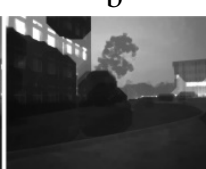

f

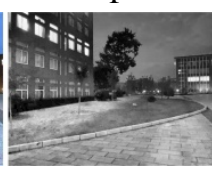

j

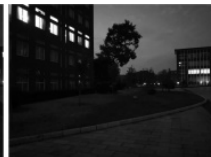

C
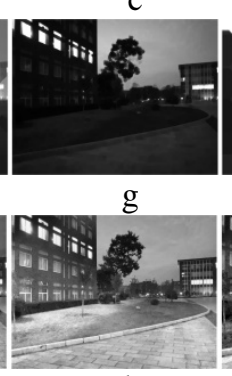

$\mathrm{k}$

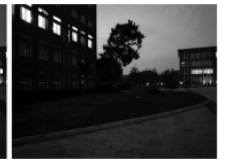

d

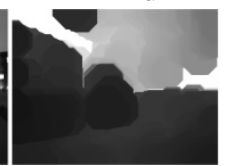

h

Fig. 6. The images of each step in the process of implementing the flowchart: (a) the source image; (b,c,d) They are the red, green and blue component images of source image; (e) is filtered result of (f) by guided filter; (f) is a fuison image which is fuison image (g) and image (h); (g) is illumination estimation processed by smaller size of closing kernel, and (h) is illumination estimation processed by larger size of closing kernel; (i) the enhanced image based on (h); (j,k,l) They are the red, green and blue component images of enhanced image. 


\section{Experiment Results}

This section will demonstrate that proposed method has excellent enhanced effect for images of low-light and uneven-light. Firstly, we evaluate the proposed method by contrast it with other enhancement methods of widely-used low-light image in both subjective and objective aspects. The subjective evaluation criterion is to observe and compare the brightness distribution and color authenticity of the enhanced image through human eyes. The objective evaluation criteria are expressed in the form of data through Grayscale histogram, average brightness and information entropy. Secondly, we enhanced the randomly selected images of low-brightness and non-uniform brightness taken by mobile phones and made subjective and objective evaluation of the enhanced results.

\subsection{Subjective comparisons}

In order to ensure the fairness of the results, the image to be enhanced used in this section of the experiment is derived from: https://dragon.larc.nasa.gov/retinex/pao/news/. To make it easy to distinguish each image, the four images are named a, b, c and d. We compare the proposed method with several methods, including Contrast-Limited Adaptive Histogram Equalization (CLAHE) [9], Multi-Scale Retinex (MSR) [10], Frankle-McCann Retinex (FMR) [11], and NASA's MSRCR [12].These methods are represented by method 1,2,3 and 4, respectively.the proposed method and source image are represented by method 5 and 0 .

Fig. 7 and Fig. 8 respectively show the overall and detailed comparison between the enhancement results of different methods. Red squares on these figures represent visible artifacts, and the green squares indicate significant color distortion.

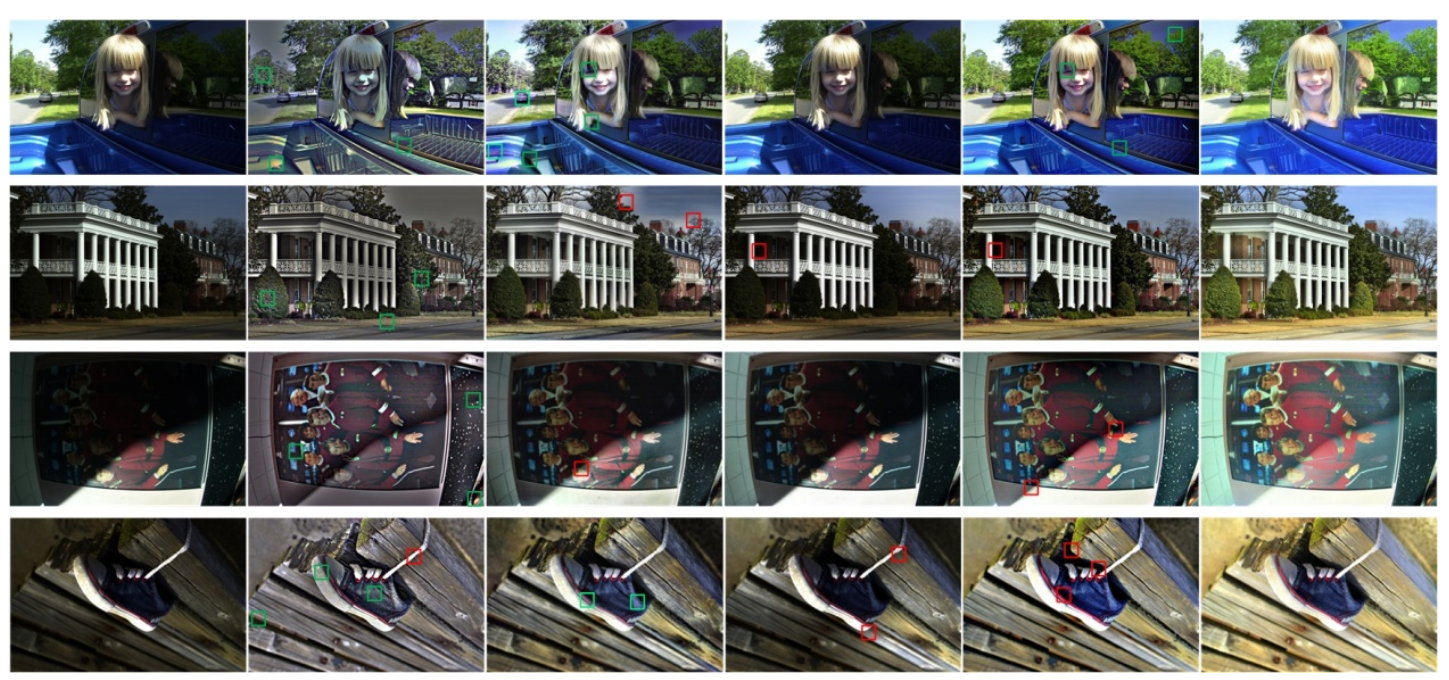

Fig. 7. Source image and result images: where, every image in Fig. 7 naming method is a two-dimensional array, which rows represents the source images of a, b, c and d; and columns represents the source image and the result image of methods 1, 2, 3, 4 and 5. According to the naming method of images and methods with above section, the images in Fig. 7 can be named (image name, method name). For instance, the image in the upper right corner can be named (a,5).

MSR attempts to enhance the overall brightness of the image by retinex, which using Gauss operators to estimate the illumination. However, the grayscale of the enhanced image will be clustered around 1 because the estimated illumination image is always close to the original image. After normalizing and stretching the narrow distribution histogram of the low-contrast 
image, MSR produce noticeable hue distortion and artifacts in local area as the continuous values of adjacent pixels are stretched apart and the red, green and blue component stretched ratios are not equal. For instance, Red and green square areas in images by method \#1 (observed in Fig. 7 and Fig. 8). In contrast, our method can generate the images of artifact-free
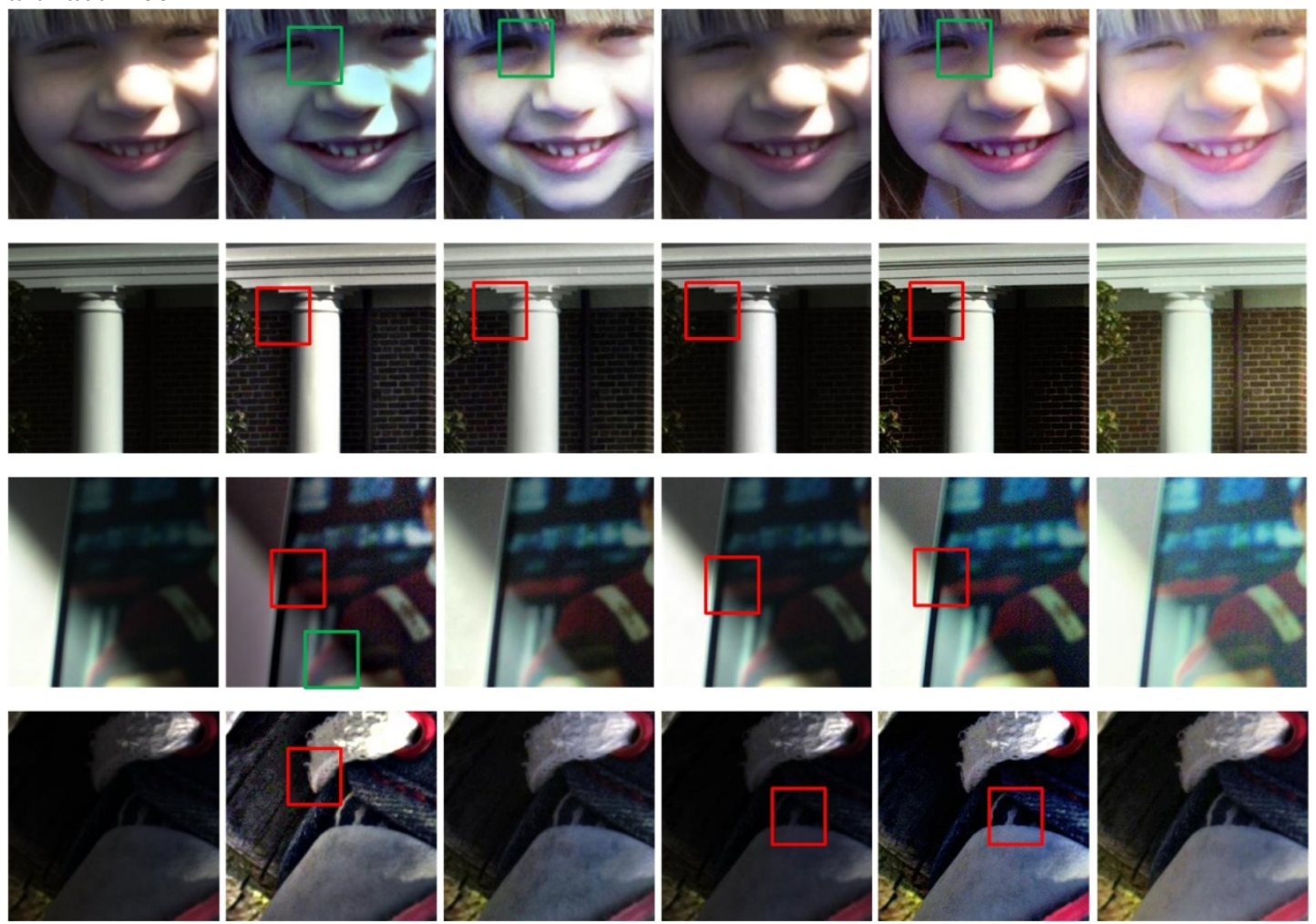

Fig. 8. The detail images of the images in Fig. 7. Its image position is consistent with Fig. 7.

and hue distortion-free, and consistent with the human visual system.

CLAHE designed to improve the lightness and contrast of images, and most of enhanced images have higher lightness and contrast. Nevertheless, it generates observable halo artifacts and hue distortion in some local area of image. Such as the sky area on the left and the face of the detail image \#a by method \#2(observed in Fig. 7 and Fig. 8). These images are not pleasing in human vision. By contrast, our method does not generate halo artifacts and tonal distortion, also produces comfortable appearance.

As discussed previously, FMR is not obvious for image enhancement with uneven brightness, as can be see in the enhanced image of method \#3 (observed in Fig. 7 and Fig. 8). Also, FMR generates observable halo artifacts in some regions. For instance, wall near white pillar in image \#b by method \#3 (observed in Fig. 8), and wood around the laces in image \#c by method \#3 (shown in Fig. 7). In fact, among all the compared methods, only the method of this paper has a strong enhancement effect on the darker side on dark side of the bright-dark boundary. For instance, in the images \#2 by method \#3 (Fig. 8)

The result images of NASA shows impressive performance overall. However, the image has subtle color deviation. Such as the tree in the mirror in the image \#1 by method \#4 (Fig. 7). And produce serious artifacts in regions on dark side of the bright-dark boundary, even darker than the source image in these regions, causes the boundary grayscale gradient to be too sharp. As can be observed in the images by method \#4 (Fig. 8). 


\subsection{Objective quality assessments}

\section{a. Corresponding histogram and table of standard deviation}

The histograms can substantially show the contrast and lightness distribution of the enhanced images. The more uniform the histogram distribution, the more uniform the image brightness distribution and the higher contrast. And illustrates that the higher the image quality.

As can be seen, in all histograms, the black lines are softest compared to lines of other colors, respectively (Fig. 9).
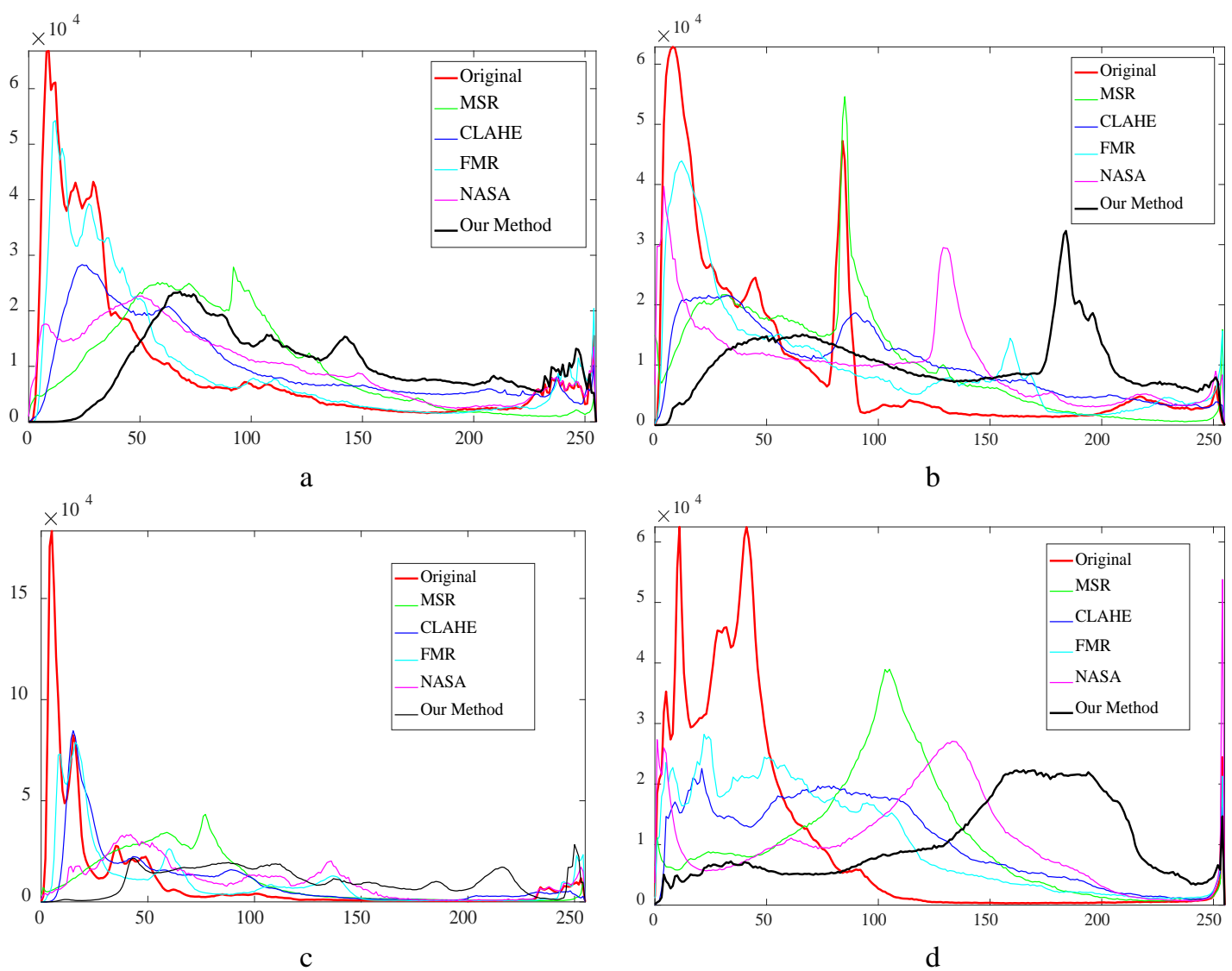

Fig. 9. Histogram of the images and methods mentioned above. where, images a, b, c, and $\mathbf{d}$ correspond to $\mathbf{a}, \mathbf{b}, \mathbf{c}$, and $\mathbf{d}$ of the source image as described above, respectively. And The red line represents the histogram of the original image, the lines of the five colors of green, blue, cyan, magenta and black represent the histograms of the enhanced images obtained by MSR, CLAHE, FMR, NASA and the method.

\section{b. Average brightness}

The average brightness is the average of the gray values of all the pixels in the image, and it can objectively evaluate the brightness of images. The gray level are all 256-order in image of this paper. In an image that conforms to the HVS, the average bightness is half of the maximum brightness in the ideal state, which is 127.5. When the average brightness of the image is around 127.5 , the brightness of the image can be considered to be visually pleasing. 
The average lightness is calculated by

$$
M=\frac{\sum_{\omega(x, y, Z) \in(X, Y, Z)} \operatorname{Img}(\omega)}{X * Y * Z}
$$

Where, $\omega$ is the pixel position, $\omega(x, y, z) \in(X, Y, Z) . X, Y, Z$ represent the maximum value of each dimension of the image, respectively, and $\operatorname{Img}(\omega)$ enotes the lightness of the pixel. The average lightness of the original image and enhancement image by each method are shown in the Table 1.

Table 1. average brightness

\begin{tabular}{|c|c|c|c|c|c|c|}
\hline & Original & MSR & CLAHE & FMR & NASA & Our Method \\
\hline a & 65.06 & 86.94 & 93.36 & 72.09 & 93.34 & $\mathbf{1 2 7 . 4 2}$ \\
\hline b & 57.01 & 77.26 & 90.35 & 76.11 & 91.45 & $\mathbf{1 2 3 . 1 7}$ \\
\hline c & 40.85 & 69.75 & 62.93 & 62.90 & 86.99 & $\mathbf{1 2 4 . 7 9}$ \\
\hline d & 41.33 & 99.02 & 85.61 & 71.60 & 106.58 & $\mathbf{1 4 0 . 3 7}$ \\
\hline average & 40.85 & 83.24 & 83.06 & 70.67 & 94.59 & $\mathbf{1 2 8 . 9 4}$ \\
\hline
\end{tabular}

Note: The data in this table comes from the image in Fig. 7. Where, the best values are represented by a bold font.

As can be seen in Table 1. The average brightness of the image enhanced by the method in this paper is closer to 127.5 compared with the results of other methods. The average brightness average increased from 40.85 to 128.94 , and the average brightness value after enhancement was 1.44. The difference from the optimal lightness value was controlled within 5 . This together with Table 1 illustrates that the method of this paper has an absolute advantage in enhancing the contrast and brightness of the image. In fact, the method of this paper can produce satisfactory results in most cases of lightness distribution.

\section{c. Information entropy}

Information entropy represents the amount of information. In the field of image processing, it is possible to objectively reflect the amount of information in the image. The larger the information entropy in the range, the more information the image contains in the area. That is, have better enhancements.

The information entropy can be easily obtained as follows:

$$
S=\sum_{n=0}^{N} I_{n} \log I_{n}
$$

where, $I_{n}$ is the probability that the image will appear grayscale n. It can be obtained by gray histogram or brute-force, it is calculated by

$$
I_{n}=\frac{H_{n}}{\sum_{n=o}^{N} H_{n}}
$$

As shown in Equation (13), $H_{n}$ represents the number of pixels with grayscale of $n$, $n \in(0,255)$. The $N$ is 255 in this paper, it's the maximum grayscale value.

In Table 2, by contrast, the purposed method has the highest enhancement of detail features, and the average information entropy has been increased from 6.8894 to 7.8810 , which is almost 1.0, better than other methods. 
Among all the compared methods, in image enhancement, the method of this paper not only has an incomparable advantage in lightness (as shown above). Moreover, the grayscale of the red, green, and blue components proportions change equally, so color distortion does not occur. This method maintains the color consistency. In contrast, other methods have caused different degrees of color distortion, respectively.

Table 2. entropy of original and enhanced images

\begin{tabular}{|c|c|c|c|c|c|c|}
\hline & Original & MSR & CLAHE & FMR & NASA & Our \\
\hline a & 7.1756 & 7.4745 & 7.7088 & 7.3224 & 7.7670 & $\mathbf{7 . 9 0 8 7}$ \\
\hline b & 7.1306 & 7.4375 & 7.7527 & 7.5529 & 7.7481 & $\mathbf{7 . 9 4 1 3}$ \\
\hline c & 6.2852 & 7.1392 & 7.1301 & 7.0562 & 7.5696 & $\mathbf{7 . 8 2 1 1}$ \\
\hline d & 6.9666 & 7.4010 & 7.6190 & 7.4663 & 7.6626 & $\mathbf{7 . 8 5 2 9}$ \\
\hline average & 6.8894 & 7.3631 & 7.5527 & 7.3495 & 7.6868 & $\mathbf{7 . 8 8 1 0}$ \\
\hline
\end{tabular}

Note: The data in this table comes from the image in Fig. 7. Where, the best values are represented by a bold font.

From the above experimental data, it can be proved that the method has excellent enhancement effect on lightness and color. The proposed method successfully enhances the visibility of low-light images while maintaining color consistent with the original image.

\section{d. Enhancing image taken by multiple scenes}

The excellent performance of the proposed method will be demonstrated in this section. And, the scene images include two parts, outdoor and indoor, including cars, buildings, flowers, close shots, distant views, etc. Both types of images contain areas that are difficult to enhance, such as extremely low brightness, uneven illumination, and point light sources. Otherwise, the method of this paper proposed the enhancement effect from the Subjective comparison and the objective evaluation consists of information entropy and average brightness, all of these illustrate the superiority of the proposed method.
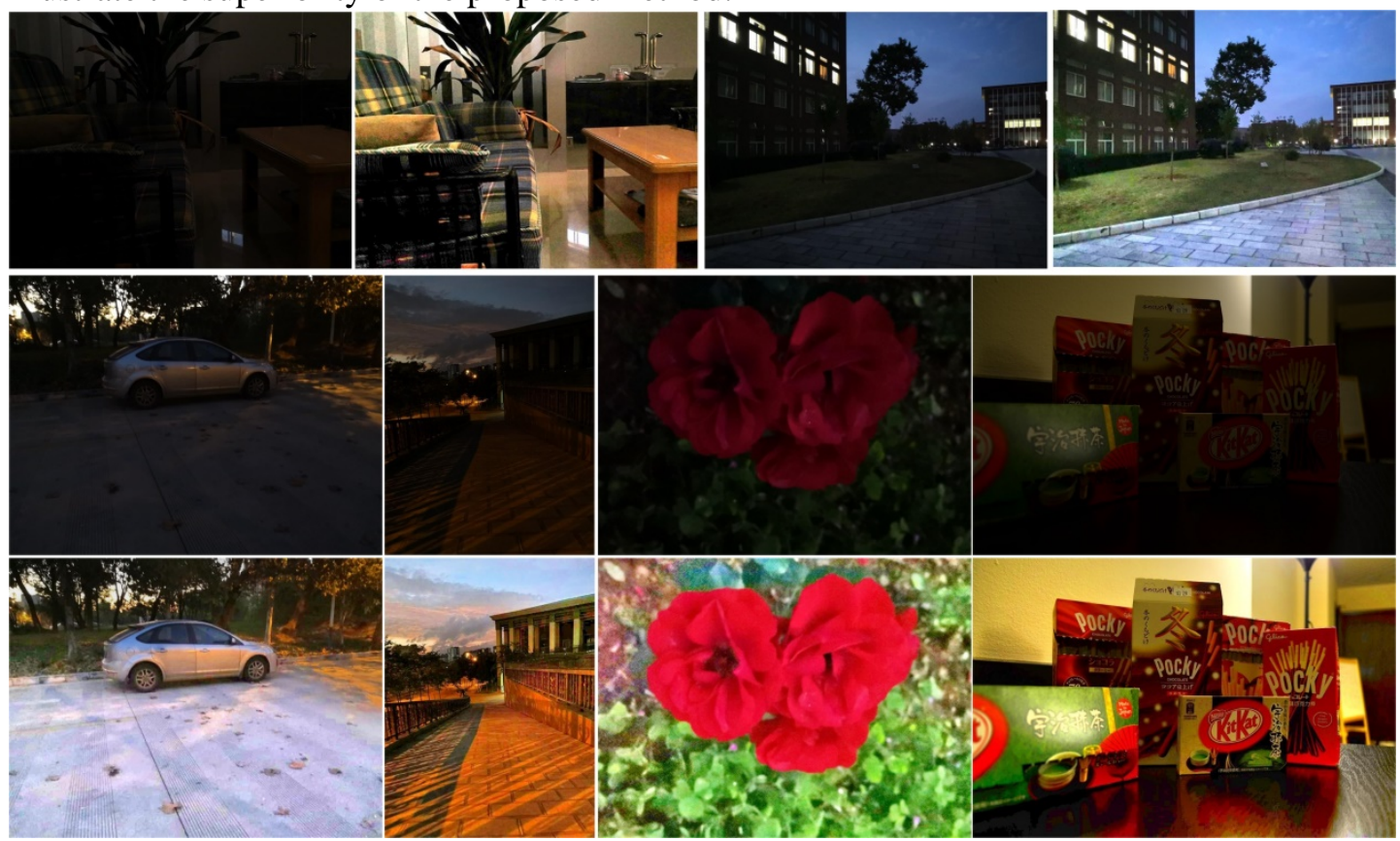

Fig. 10. Original image and the enhanced image of proposed method. The enhanced image is on the right and below the original image, respectively. 
The following two tables will list the information entropy and average brightness of the original and enhanced images above.

Where, in Table 3 and Table 4, the average information entropy of the image increased from 5.5651 to 7.3854, an increase of 1.8203; the average value of the average gray value of the image increased from 22.56 to 100.03, an increase of 77.47, and the value after the increase was closed to the ideal gray average of 127.5. These experimental results can prove that method of this paper has excellent effects on low-light images.

Table 3. Entropy of original and enhanced images

\begin{tabular}{|c|c|c|c|c|c|c|}
\hline Image & $\mathbf{a}$ & b & C & d & e & f \\
\hline Original & 4.7701 & 5.4386 & 5.5491 & 5.8633 & 5.3734 & 6.3963 \\
\hline Enhanced & 6.8635 & 6.7667 & 7.7007 & 7.5245 & 7.6706 & 7.7846 \\
\hline \multicolumn{2}{|c|}{ Original Average } & \multicolumn{2}{|l|}{5.5651} & ced Ave I & & \\
\hline
\end{tabular}

Table 4. Average lightness of source image and enhanced image

\begin{tabular}{|c|c|c|c|c|c|c|}
\hline Image & a & b & c & d & e & f \\
\hline Original & 10.65 & 21.07 & 26.76 & 22.90 & 16.38 & 37.57 \\
\hline Enhanced & 71.34 & 75.59 & 136.96 & 100.73 & 109.33 & 106.21 \\
\hline Original Average & $\mathbf{2 2 . 5 6}$ & \multicolumn{2}{|c|}{ Enhanced Average } & $\mathbf{1 0 0 . 0 3}$ \\
\hline
\end{tabular}

\section{e. Compared with the same type of deep learning method}

In this section, we will use our method to compare with the latest methods of deep learning. Although deep learning has unparalleled advantages in many respects compared to traditional algorithms, the advantages of deep learning in this field are not well reflected. For example, although it can be trained to get a good filter, it still needs manual work to get the optimal results, and the characteristics that are easily over-enhanced greatly limit its advantages. The following are images and explanation by comparing the method of this paper and LSD(Learning to See in the Dark) [32] .

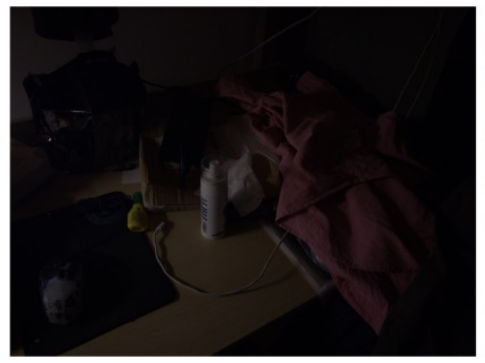

a

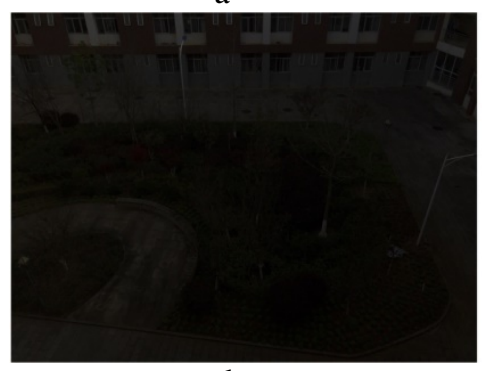

d

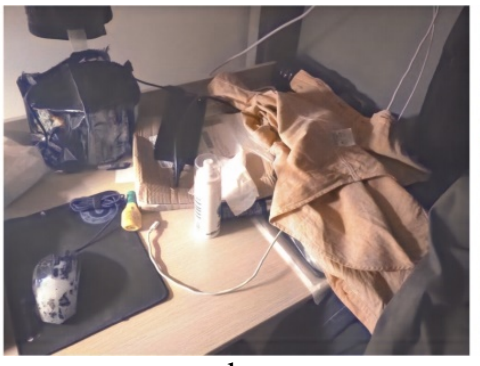

b

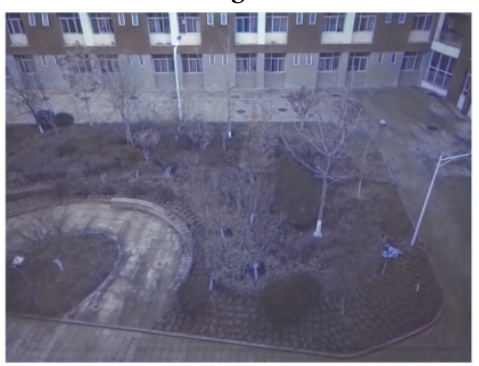

e

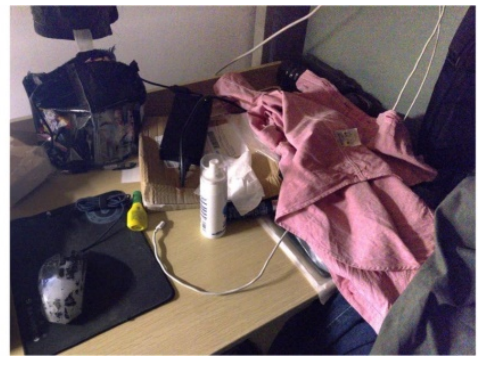

C

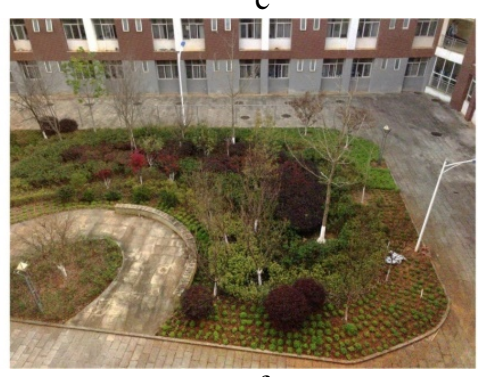

$\mathrm{f}$

Fig. 11. Comparison of the purposed method and LSD. a,d: natural image; b,e: the best result of LSD, ratio $=100$; c,f: the result of our method, The parameters are consistent with the above mentioned. 

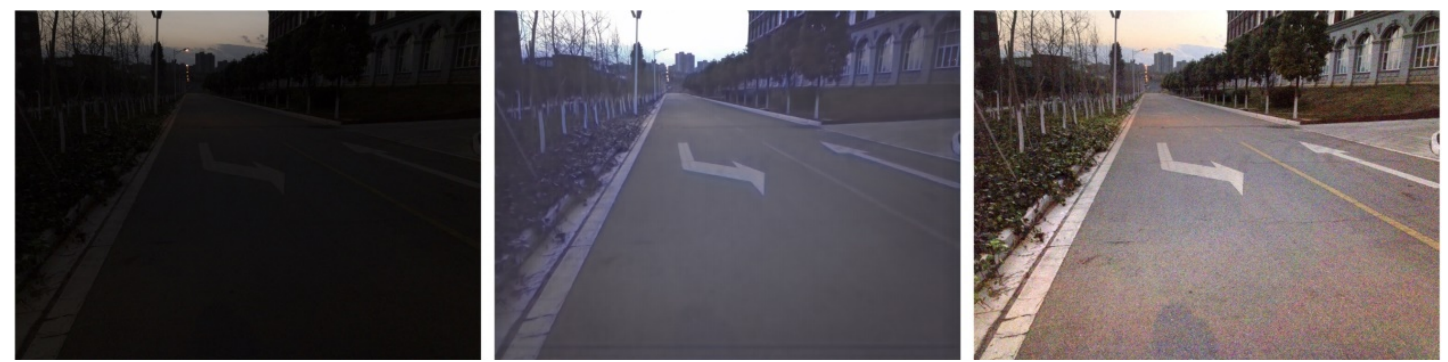

Fig. 12. Enhanced image of low-illumination and high-noise.

As can be see, In Fig. 11, We can see the problem clearly of the result of LSD (\#b and \#e). On the first, It produces a non-benign color shift compared to the original image (image a and d), such as the pink shirt on the table, Red flower and green tree in the yard. Secondly, the image is excessively smooth and duplicate artificial edges are produced, such as the area below in the image \#b and \#e (Fig. 11); Last but not least, LSD has a lot of limitations. It can be proved from experiments that it is only suitable for images with approximate illumination environment. Otherwise, it is easy to overexpose or the dark part is not obvious, and the final 'good' image needs 'people' find out. For instance, a lot of details are lost around the white bottle due to overexposure. In our methods, noise is well suppressed during the enhancement process, and will not have too much impact on visual information, in \#c and \#f (Fig. 11). More importantly, the color information is completely preserved. However, this method only suppresses noise, instead of denoising, as shown in Fig. 12.

\section{Conclusion}

In this paper, For lacking of light, uneven illumination, weather changes, relative motion, overexposure and other factors, the visual quality of acquisition image is reduced, and leads corresponding challenge on subsequent applications. For different imaging condition, image enhancing method can't be generalized. Image enhancement is a challenging problem in image processing, especially for low-light image. This paper aims to survey related research work, analyze them, and experiment them, and finally proposed a robust and comprehensive enhancement method from several aspects. First, the method of bright channel is adopted to estimation the illumination intensity for keep color constancy. Second, for eliminating offsets in estimating result with bright channel, morphological closing operation to correct. Furthermore, a multi-scale closing operation is adopted for eliminating the fabricating edges and noise amplification. At last, in order to avoiding the haloes and artifacts presented in enhancing result for gradient information lost in previous step, guided filtering is used to deal with result of multi-scale closing operation. The guided image is initial estimating illumination estimation map by brigth channe. The proposed comprehensive enhancement method not only has effective enhancement effect on low illumination image,more important is to main color constancy and natural, avoid false edge, artifacts,halos and resist noise amplification. Stepwise experiments, objective and subjective evaluation all prove that the proposed method effective. 


\section{Acknowledgments}

The authors would like to thank the anonymous reviewers for their detailed review and constructive comments. This work was partially supported by National Natural Science Foundation of China (No. 11773012)

\section{References}

[1] Raman Maini and Himanshu Aggarwal, "A Comprehensive Review of Image Enhancement Techniques,” Journal of Computing, vol. 2, no. 3, pp. 8-13, March, 2010. Article (CrossRef Link).

[2] Retinex: Wikis. www.thefullwiki.org/Retinex

[3] Land E H, “Recent advances in Retinex theory," Vision Research, vol. 26, no. 1, pp. 7-21, 1986. Article (CrossRef Link).

[4] D. J. Jobson, Z. Rahman and G. A. Woodell, "Properties and performance of a center/surround retinex," IEEE Transactions on Image Processing, vol. 6, no. 3, pp. 451-462, Mar, 1997. Article (CrossRef Link).

[5] MU Qi, WEI Yanyan, etc, "Research on the improved retinex algorithm for low illumination image enhancement," Journal of Harbin Engineering University. vol.39, No.12, pp. 2001-2010, December, 2018. Article (CrossRef Link).

[6] Gonzalez R C, Woods R E, Eddins S L, Digital image processing using MATLAB, Pearson-Prentice-Hall, Upper Saddle River, New Jersey, 2004.

[7] Guo X, Li Y, Ling H, "LIME: Low-light image enhancement via illumination map estimation," IEEE Transactions on Image Processing, vol. 26, No. 2, pp. 982-993, Feb. 2017. Article (CrossRef Link).

[8] He K, Sun J, Tang X, “Guided image filtering,” IEEE transactions on pattern analysis \& machine intelligence, vol. 35, No. 6, pp. 1397-1409, June, 2013. Article (CrossRef Link).

[9] Zuiderveld K, “Contrast limited adaptive histogram equalization,” Graphics gems IV. Academic Press Professional, Inc., pp. 474-485, 1994. Article (CrossRef Link).

[10] Rahman Z, Jobson D J, Woodell G A, "Retinex processing for automatic image enhancement," Journal of Electronic imaging, Vol. 13, No. 1, pp. 100-111, Jan. 2004. Article (CrossRef Link).

[11] Funt B V, Ciurea F, McCann J J, "Retinex in matlab ${ }^{\mathrm{tm}}$," Journal of electronic imaging, Vol. 13, No. 1, pp. 48-58, Jan. 2004. Article (CrossRef Link).

[12] NASA, Retinex Image Processing, 2001. [Online]. Available: https://dragon.larc.nasa.gov/retinex/pao/news.

[13] Fu X, Sun Y, LiWang M, et al., "A novel retinex based approach for image enhancement with illumination adjustment," in Proc. of 2014 IEEE International Conference on Acoustics, Speech and Signal Processing (ICASSP), pp. 1190-1194, 2014. Article (CrossRef Link).

[14] Matin F, Jeong Y, Kim K, et al., "Color Image Enhancement Using Multiscale Retinex Based on Particle Swarm Optimization Method," in Proc. of Journal of Physics: Conference Series. Vol. 960 No. 1, pp. 012-026, 2018. Article (CrossRef Link).

[15] Hsu M C, Lo Y S, Lin C H, "Retinex image enhancement based on exposure fusion," in Proc. of 2018 3rd International Conference on Intelligent Green Building and Smart Grid (IGBSG). IEEE, pp. 1-5, 2018. Article (CrossRef Link).

[16] Li M, Liu J, Yang W, et al., "Structure-Revealing Low-Light Image Enhancement Via Robust Retinex Model," IEEE Transactions on Image Processing, Vol. 27, No. 6, pp. 2828-2841, 2018. Article (CrossRef Link).

[17] Ahn H, Keum B, Kim D, et al., "Adaptive local tone mapping based on retinex for high dynamic range images," in Proc. of 2013 IEEE International Conference on Consumer Electronics (ICCE), pp. 153-156, 2013. Article (CrossRef Link).

[18] Wang Y, Wang H, Yin C, et al., "Biologically inspired image enhancement based on Retinex," Neurocomputing, Vol. 177, pp. 373-384, Feb. 2016. Article (CrossRef Link). 
[19] Tang S, Dong M, Ma J, et al., "Color image enhancement based on retinex theory with guided filter,” in Proc. of 2017 29th Chinese Control And Decision Conference (CCDC), pp. 5676-5680 2017. Article (CrossRef Link).

[20] Zhang Y, Huang W, Bi W, et al., "Colorful image enhancement algorithm based on guided filter and Retinex,” in Proc. of Signal and Image Processing (ICSIP), IEEE International Conference on. IEEE, pp. 33-36, 2016. Article (CrossRef Link).

[21] Fu X, Zeng D, Huang Y, et al., “A fusion-based enhancing method for weakly illuminated images," Signal Processing, Vol. 129, pp. 82-96, 2016. Article (CrossRef Link).

[22] Li L, Wang R, Wang W, et al., “A low-light image enhancement method for both denoising and contrast enlarging,” in Proc. of Image Processing (ICIP), 2015 IEEE International Conference on. IEEE, pp. 3730-3734, 2015. Article (CrossRef Link).

[23] Zotin A, "Fast Algorithm of Image Enhancement based on Multi-Scale Retinex," Procedia Computer Science, Vol. 131, pp. 6-14, 2018. Article (CrossRef Link).

[24] Xiao J, Peng H, Zhang Y, et al., "Fast image enhancement based on color space fusion," Color Research \& Application, Vol. 41, No. 1, pp. 22-31, 2016. Article (CrossRef Link).

[25] Kim S E, Jeon J J, Eom I K, "Image contrast enhancement using entropy scaling in wavelet domain,” Signal Processing, Vol. 127, pp. 1-11, 2016. Article (CrossRef Link).

[26] Park S, Yu S, Moon B, et al., "Low-light image enhancement using variational optimization-based retinex model,” IEEE Transactions on Consumer Electronics, Vol. 63, No. 2, pp, 178-184, 2017. Article (CrossRef Link).

[27] Park S, Moon B, Ko S, et al., "Low-light image restoration using bright channel prior-based variational Retinex model,” EURASIP Journal on Image and Video Processing, Vol. 2017, pp. 44, 2017. Article (CrossRef Link).

[28] Gao Y, Hu H M, Li B, et al., "Naturalness Preserved Nonuniform Illumination Estimation for Image Enhancement Based on Retinex,” IEEE Transactions on Multimedia, Vol. 20, No.2, pp, 335-344 2018. Article (CrossRef Link).

[29] Jiang B, Woodell G A, Jobson D J, "Novel multi-scale retinex with color restoration on graphics processing unit,” Journal of Real-Time Image Processing, Vol. 10, No. 2, pp. 239-253, 2015. Article (CrossRef Link).

[30] Fu Q, Jung C, Xu K, "Retinex-based perceptual contrast enhancement in images using luminance adaptation,” IEEE Access, Vol. 6, pp. 61277-61286, 2018. Article (CrossRef Link).

[31] Zhang S, Wang T, Dong J, et al., "Underwater image enhancement via extended multi-scale Retinex,” Neurocomputing, Vol. 245, pp. 1-9, 2017. Article (CrossRef Link).

[32] Chen Chen, Qifeng Chen, Jia Xu, Vladlen Koltun, "Learning to See in the Dark," in Proc. of The IEEE Conference on Computer Vision and Pattern Recognition (CVPR), pp. 3291-3300, 2018. Article (CrossRef Link).

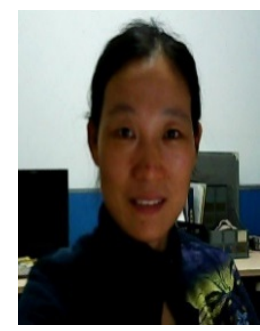

Cuiyin Liu received her Ph.D. in college of Computer Science at SiChuang University, Chengdu,China,in2013. She is currently associate professor at Computer Center at Kunming University of Science and Technology, Kunming, China. Her research interests focus on image fusion, image enhancement, image registration and astronomical observation image processing. Email:liucuiyin@kmust.edu.cn.

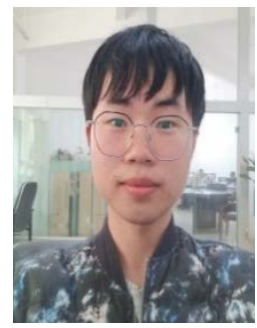

Fanglong Wu is currently master student in Kunming University of Science and Technology, Kunming, China. His current research interest is image enhancement especially in low-light image, noisy image and blurred image. Eamial: fanglongwu.stu@gmail.com. 